\title{
THE ORIGIN OF LATE ARCHAEAN GRANITOIDS IN THE SUKUMALAND GREENSTONE BELT OF NORTHERN TANZANIA: GEOCHEMICAL AND ISOTOPIC CONSTRAINTS.
}

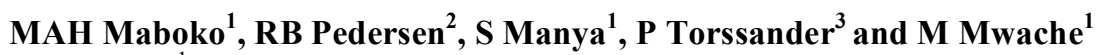 \\ ${ }^{1}$ Department of Geology, University of Dar es Salaam \\ P.O. Box 35052, Dar es Salaam, Tanzania \\ *Corresponding author (email: mmaboko@hotmail.com) \\ ${ }^{2}$ Department of Geology, University of Bergen \\ Allegt. 41, N-5007 Bergen, Norway \\ ${ }^{3}$ Department of Geology and Geochemistry \\ Stockholm University \\ S-106 91 Stockholm, Sweden
}

\begin{abstract}
Granitoids intruding the late Archaean sequences of the Sukumaland Greenstone Belt of northern Tanzania belong to two distinct geochemical suites. Suite 1 is characterised by $\mathrm{Na}_{2} \mathrm{O} / \mathrm{K}_{2} \mathrm{O}>1$ (1.04 - 4.67), high $\mathrm{Sr} / \mathrm{Y}(56$ - 204) and Ba/Rb ratios $(6.1$ - 27.1) and low Rb/Sr ratios (0.08 $0.25)$. The rocks are enriched in $\mathrm{Sr}(405-1264 \mathrm{ppm})$ and depleted in $\mathrm{Yb}(0.17-0.93 \mathrm{ppm})$ and $\mathrm{Rb}$ $(56-132 \mathrm{ppm})$. On chondrite-normalised REE diagrams, the rocks display highly fractionated patterns characterised by relative LREE enrichment $\left((\mathrm{La} / \mathrm{Yb})_{N}=23-128\right.$ and $(\mathrm{Gd} / \mathrm{Yb})_{N}=3.10-$ $8.54)$ and lower concentrations of the HREE $\left(Y b_{N}=0.80-4.45\right)$. On primitive mantle-normalised spidergrams, $\mathrm{Nb}$ and $\mathrm{Ti}$, together with $\mathrm{P}$ and $\mathrm{Y}$ are depleted relative to adjacent elements. The major and trace element characteristics of Suite 1 are comparable to those of typical Archaean TTG suites and High Silica Adakites (HSA). Suite 2 granitoids are characterised by $\mathrm{Na}_{2} \mathrm{O} / \mathrm{K}_{2} \mathrm{O}<$ 1, low $\mathrm{Sr} / \mathrm{Y}(2.80-41.7)$ and $\mathrm{Ba} / \mathrm{Rb}(0.40-8.91)$ ratios and high $\mathrm{Rb} / \mathrm{Sr}(0.30-6.27)$ ratios. Suite 2 is also characterised by low $\mathrm{Sr}(53-326 \mathrm{ppm})$ and high $\mathrm{Rb}(40-365 \mathrm{ppm})$ and $\mathrm{Yb}(0.44-1.36$ ppm) contents. Compared to Suite 1, Suite 2 rocks display less fractionated REE patterns $\left((L a / Y b)_{N}=15-86\right.$ and $\left.(G d / Y b)_{N}=1.73-6.74\right)$ and are characterised by higher concentrations of the HREE $(Y b N=2.1-6.5)$. On primitive mantle-normalised spidergrams, Suite 2 samples, like those of Suite 1, show relative depletion in $\mathrm{Th}, \mathrm{Nb}$ and Ti, together with $\mathrm{P}$ and $Y$ relative to adjacent elements. Sm-Nd mean crustal residence ages for both suites are indistinguishable and range between 2470 and $2720 \mathrm{Ma}$ with a mean of $2610 \pm 35 \mathrm{Ma}$ (2 SE), similar to the emplacement age of $2620 \pm 40 \mathrm{Ma}$. The granitoids are interpreted to have formed by partial melting at the base of a late Archaean thickened sub-arc basaltic crust. Melting to form the Suite 1 granitoids occurred in the eclogite stability field whereas Suite 2 formed by melting at shallower depth in the garnet amphibolite stability field.
\end{abstract}

\section{INTRODUCTION}

Processes of Archaean continental crust formation continue to generate a great deal of interest among earth scientists (Drummond and Defant 1990, Smithies 2000). In particular, much effort has been directed at comparing processes responsible for the formation of Archaean granitegreenstone terranes with those operating in Phanerozoic tectonic settings (Drummond and Defant 1990, Smithies 2000). Central in the debate has been the origin of granitic rocks of the tonalite-trondhjemitegranodiorite (TTG) suite that form a major component of many Archaean terranes. Some workers have interpreted the genesis of TTG in terms of slab melting processes akin to those responsible for the formation of Phanerozoic adakites (Drummond and Defant 1990, Martin 1999, Martin et al. 2005) whose formation is only confined to those areas where, as in the Archaean, young 
Maboko et al.- The origin of late archaean granitoids in the Sukumaland ...

and warm easy to melt oceanic lithosphere is subducted (Drummond and Defant 1990). Other workers, however, urgue that unlike most Cenozoic adakites, Archaean TTG show no evidence of interaction of the parental magmas with mantle peridotite which is reflected in low $\mathrm{SiO}_{2}$ contents (as low as $58 \%$ ), high $\mathrm{Mg}$ numbers (as high as 68 ) and high concentrations of $\mathrm{Ni}$ and $\mathrm{Cr}$ (Smithies 2000). Thus, they postulate a model for TTG generation in which partial melting of hydrated basalt occurs at the base of magmatically and/or tectonically thickened crust. A similar tectonic setting has been postulated for Phanerozoic Na-rich granitoids that share many geochemical and petrological similarities with Archaean TTG (e.g. Atherton and Petford 1993).
The Tanzania craton of eastern Africa consists of $2.5-2.8 \mathrm{Ga}$ granitoids, intruding and flanking a number of greenstone belts in northern Tanzania and western Kenya (Borg and Shackleton 1997). Although forming an important component of the Archaean crust of East Africa, there has been no modern geochemical investigation of the granitoids and their role in the growth and stabilisation of the Tanzania craton. This paper, therefore, presents major and trace element geochemical as well as $\mathrm{Nd}$ isotopic data of granitoids from the Sukumaland Greenstone Belt (SGB) sampled along a $\sim 100 \mathrm{~km}$ long transect trending roughly north south from Geita to Kahama (Fig. 1). The data are used to constrain the petrogenesis and tectonic setting of the granitoids.

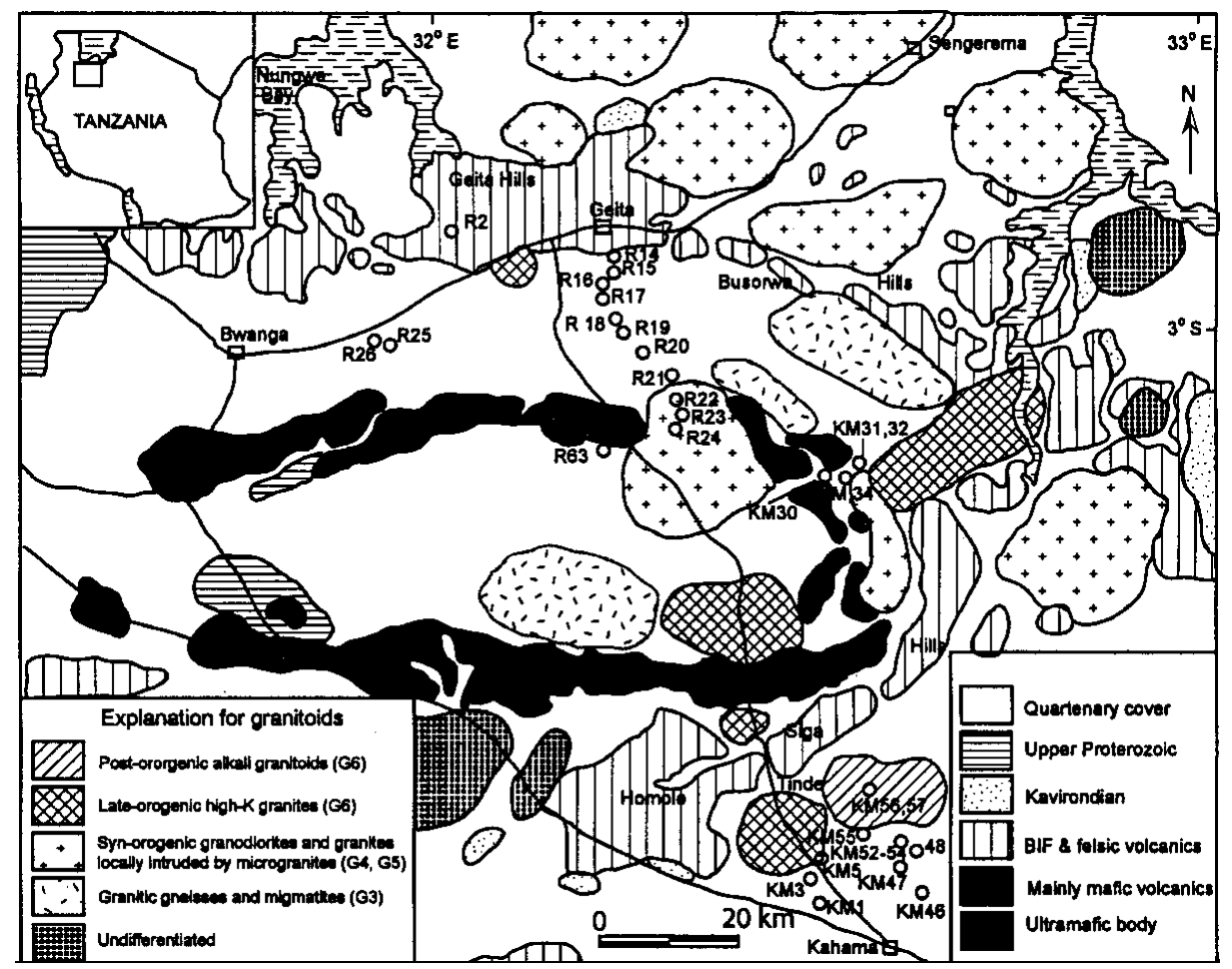

Figure 1: Geological map of the Sukumaland Greenstone Belt (SGB) showing sampling localities (as circles and numbers). The frame in the inset map shows the location of the Sukumaland greenstone Belt. 


\section{Geological background}

In the area south of Lake Victoria (Fig. 1), the Nyanzian Belt is represented by rocks of the Sukumaland Greenstone Belt (Borg et al. 1990). The Sukumaland Greenstone Belt consists of gabbro, pillow basalt and subordinate felsic flows and pyroclastics overlain by a sequence composed predominantly of BIF, felsic pyroclastics and flows and carbonaceous shales metamorphosed to the greenschist facies and locally to the middle amphibolite facies. The relative proportion by volume of the volcanic rocks has been estimated as $40 \%$ mafic, $30 \%$ andesite and $20 \%$ felsic whereas the ratio of sedimentary to igneous rocks has been estimated at 1:8 (Borg and Shackleton 1997). The maximum preserved thickness of the greenstone sequence as estimated using geophysical methods is $5-7 \mathrm{~km}$ (Borg and Shackleton 1997).

Numerous granitoids intrude and flank the Nyanzian Greenstone Belts. Borg and Shackleton (1997) estimated a ratio of granite to greenstone in the belt of $4: 1$ by volume. Earlier studies by Grantham et al. (1945) distinguished four different generations of granitoids in the Tanzania craton on the basis of their chemical and petrographic composition and by their tectonic location in relation to the greenstone belts. The first generation (G3) includes granitic gneisses and migmatites, which occupy structurally lower positions than the greenstones. The second generation comprises syn-orogenic granite and granodiorite (G4 and G5) whereas postorogenic alkali granites and syenite (G6) comprise the youngest generation. Barth (1990) who observed that it was unlikely that a single intrusive differentiation sequence exists for the granitoids in the Tanzania craton proposed an alternative classification in which he subdivided the rocks into a syn-orogenic and a latekinematic (late or post-orogenic) intrusive cycle. The syn-orogenic cycle comprises of migmatites, foliated and porphyroblastic granites, biotite-hornblende granites, trondhjemites, granodiorites, tonalites, monzonites and quartz diorites with an excess of $\mathrm{Na}$ over K (Barth 1990). The latekinematic cycle comprises biotite granite, microgranite, alaskite, leucogranites and feldspar porphyries (Barth 1990). Thus, this scheme groups the G3 to G5 granites of Grantham et. al. (1945) into a single synorogenic suite while maintaining the G6 granites as a distinct post-orogenic suite.

The Na-rich granitoids (equivalent to the G3 suite of Grantham et al. 1945), which intrude and flank the greenstone sequences in the Geita area, have yielded a $\mathrm{Rb}-\mathrm{Sr}$ whole rock isochron age of $2620 \pm 30 \mathrm{Ma}$ (initial ${ }^{87} \mathrm{Sr} /{ }^{86} \mathrm{Sr}$ ratio $=0.7017 \pm 4$ ) reported by Bell and Dodson (1981). The supracrustal assemblages in the Sukumaland Greenstone Belt have yielded a $\mathrm{Sm}-\mathrm{Nd}$ isochron age of $2823 \pm 44 \mathrm{Ma}$ reported by Manya and Maboko (2003) for the Nyanzian metabasalts from Rwamagaza. Borg and Krogh (1999) reported single zircon U-Pb ages of $2780 \pm 3$ and $2808 \pm 3$ Ma for the Nyanzian rhyolitic pyroclastic rocks from the outer arc of the SGB north to north-west of Kahama.

\section{MATERIALS AND METHODS}

Thirty one granitoid samples collected along a $\sim 100 \mathrm{~km}$ long N-S trending transect from Geita to Kahama (Fig. 1) were analysed for major elements at the ACME Analytical Laboratories in Vancouver, Canada. Trace elements for a batch of 16 samples were also analysed at the ACME Laboratories. For the major elements and $\mathrm{Ba}$, aliquots of the pulverised samples, mixed with $\mathrm{LiBO}_{2}$ flux, were melted prior to acid digestion and analysis by ICP-OES. All the other elements were analysed using ICP-MS following $\mathrm{LiBO}_{2}$ fusion and acid digestion. Trace elements for the remaining 15 samples were analysed at the Geochemical Laboratory of the University of Dar es Salaam using a Varian Vista MPX ICP-OES as described in Messo (2004). The analytical reproducibility, expressed as the Relative Standard Error of the Mean, obtained from 
Maboko et al.- The origin of late archaean granitoids in the Sukumaland ...

repeated measurement of the USGS Reference Material W2 is better than $8 \%$ for most elements and is typically $\sim 2.5 \%$ ( $\mathrm{n}=$ 10) for most elements except for $\mathrm{Er}, \mathrm{Gd}, \mathrm{Pr}$ and $\mathrm{Tm}$ which are accurate to within 10.8, $19.2,20.0$ and $10.5 \%$ respectively of their respective certified values.

Fifteen samples were also analysed for $\mathrm{Nd}$ isotopic composition as well as $\mathrm{Nd}$ and $\mathrm{Sm}$ concentrations on a Finnigan MAT 262 thermal ionisation mass spectrometer at the Laboratory for Isotope Geology of the Department of Geology, University of Bergen in Norway. $\mathrm{Sm}$ and $\mathrm{Nd}$ were separated by specific-extraction chromatography using a modified version of the method described by Richard et al. (1976). Sm and Nd were loaded onto a double filament and analysed in static and dynamic modes, respectively. $\mathrm{Nd}$ isotopic ratios were corrected for mass fractionation using a ${ }^{146} \mathrm{Nd} /{ }^{144} \mathrm{Nd}$ value of 0.7219 . Sm and $\mathrm{Nd}$ concentrations were determined using a mixed ${ }^{150} \mathrm{Nd}^{-{ }^{149} \mathrm{Sm}}$ spike. Repeated measurements of Johnson and Mattey $\mathrm{NdO}_{3}$ standard at the time of the analyses yielded a mean ${ }^{143} \mathrm{Nd} /{ }^{144} \mathrm{Nd}$ ratio of $0.511102 \pm 5$ $(2 \sigma)$.

\section{RESULTS}

The major and trace element composition of the samples is summarised in Table 1. On the basis of the geochemical data, the granitoids can be subdivided into two distinct geochemical suites. Suite 1 consists of 15 samples with $\mathrm{Na}_{2} \mathrm{O} / \mathrm{K}_{2} \mathrm{O}>1$ (1.04 4.67). In the area near Kahama, this suite consists mostly of foliated granitoids. Further north, however, granitoids of this suite tend to lack the gneissic foliation. According to the molecular normative $\mathrm{Ab}-$ An - Or classification scheme of Barker (1979), the rocks plot in the granite trondhjemite fields with most samples straddling the boundaries between trondhjemite, tonalite and granodiorite (Fig. $2)$. The rocks are enriched in $\mathrm{Al}_{2} \mathrm{O}_{3}$ (13.917.0, mean $=15.5$ weight \%) with $\mathrm{SiO}_{2}$ contents ranging between 66.6 and $74.5 \%$ $($ mean $=70.5)$. The combination of high $\mathrm{Al}_{2} \mathrm{O}_{3}$ and relatively high $\mathrm{SiO}_{2}$ contents indicates that the rocks are high Altrondhjemites (Barker 1979) and are compositionally similar to the High Silica Adakites (HSA) of Martin et al. (2005).

Suite 1 samples are characterised by high $\mathrm{Sr} / \mathrm{Y}(56-204$, mean $=124)$, high $\mathrm{Ba} / \mathrm{Rb}$ $(6.1-27.1$, mean $=12.9)$ and low $\mathrm{Rb} / \mathrm{Sr}$ $(0.08-0.25$, mean $=0.16)$ ratios. The rocks are also enriched in $\mathrm{Sr}(405-1264 \mathrm{ppm}$, mean $=634 \mathrm{ppm})$ and depleted in $\mathrm{Yb}(0.17-$ $0.93 \mathrm{ppm}$, mean $=0.40 \mathrm{ppm})$ and $\mathrm{Rb}(56-$ $132 \mathrm{ppm}$, mean $=92 \mathrm{ppm})$. On chondritenormalised REE diagrams (normalising values after Boynton 1984), the Suite 1 rocks display highly fractionated patterns (Fig. 3) as reflected by $(\mathrm{La} / \mathrm{Yb})_{\mathrm{N}}$ values of between 23 and 128 (mean $=71$, the subscript $\mathrm{N}$ refers to chondrite normalised values) and $(\mathrm{Gd} / \mathrm{Yb})_{\mathrm{N}}$ values between 3.10 and 8.54 (mean $=5.64)$. All samples, except R17 which has no anomaly, are characterised by slightly negative $\mathrm{Eu}$ anomalies $\left(\mathrm{Eu} / \mathrm{Eu}^{*}=0.52-0.97\right.$, mean $=$ $0.76)$. The low $\mathrm{Yb}$ contents $\left(0.8<\mathrm{Yb}_{\mathrm{N}}<\right.$ 4.5 , mean $=1.89$ ) and strongly fractionated REE patterns are also typical of other Archaean TTG suites and contrasts with the less fractionated $\left((\mathrm{La} / \mathrm{Yb})_{\mathrm{N}}<20\right)$ and higher $\mathrm{Yb}$ contents of post-Archaean granitic rocks (Drummond and Defant 1990). On primitive mantle-normalised spidergrams (normalising values after McDonough and Sun 1995), the High Field Strength Elements (HFSE) Nb and $\mathrm{Ti}$, together with $\mathrm{P}$ and $\mathrm{Y}$ are depleted whereas $\mathrm{Zr}$ and $\mathrm{Hf}$ are enriched relative to adjacent elements. $\mathrm{Sr}$ is, however, enriched relative to $\mathrm{Ce}$ and $\mathrm{Nd}$ (Fig. 4). 
Tanz. J. Sci. Vol 32(1) 2006

Table 1: $\quad$ Major (in oxide weight \%) and trace (in ppm) element composition of granitoids from the Sukumaland Greeenstone Belt

SUITE 1 GRANITOIDS

\begin{tabular}{|c|c|c|c|c|c|c|c|c|c|c|c|c|c|c|c|c|}
\hline Sample & R 2 & R 14 & R 15 & R 16 & R 17 & R 18 & R 19 & R 20 & R 21 & R 21* & R 26 & KM30 & KM31 & KM32 & KM34 & KM46 \\
\hline Classification & G3 & G3 & G3 & G3 & G3 & G3 & G3 & G3 & G3 & G3 & G3 & G3 & G3 & G3 & G3 & G3 \\
\hline $\mathrm{SiO}_{2}$ & 66.63 & 68.68 & 69.86 & 69.84 & 68.16 & 71.51 & 71.76 & 72.22 & 70.42 & 70.41 & 72.88 & 67.92 & 74.46 & 70.47 & 70.44 & 72.95 \\
\hline $\mathrm{TiO}_{2}$ & 0.3 & 0.37 & 0.28 & 0.38 & 0.27 & 0.23 & 0.15 & 0.15 & 0.27 & 0.26 & 0.24 & 0.41 & 0.14 & 0.33 & 0.32 & 0.09 \\
\hline $\mathrm{Al}_{2} \mathrm{O}_{3}$ & 16.25 & 16.36 & 15.22 & 15.38 & 17.01 & 14.95 & 15.2 & 15.08 & 15.39 & 15.39 & 14.47 & 16.53 & 13.94 & 15.89 & 15.45 & 14.85 \\
\hline $\mathrm{Fe}_{2} \mathrm{O}_{3}$ tot & 3.33 & 2.53 & 2.51 & 2.62 & 2.55 & 2.08 & 1.35 & 1.68 & 2.24 & 2.22 & 2.13 & 3.12 & 1.09 & 2.29 & 2.09 & 0.87 \\
\hline $\mathrm{MnO}$ & 0.08 & 0.03 & 0.03 & 0.04 & 0.03 & 0.03 & 0.03 & 0.03 & 0.03 & 0.03 & 0.04 & 0.05 & 0.02 & 0.03 & 0.02 & 0.01 \\
\hline $\mathrm{MgO}$ & 1.03 & 0.84 & 0.54 & 0.65 & 0.72 & 0.4 & 0.27 & 0.32 & 0.53 & 0.54 & 0.4 & 1.22 & 0.27 & 0.73 & 0.67 & 0.3 \\
\hline $\mathrm{CaO}$ & 3.07 & 3.02 & 2.15 & 2.08 & 3.15 & 1.71 & 1.64 & 1.76 & 2.09 & 2.1 & 1.64 & 3.51 & 1.32 & 2.98 & 2.01 & 2.14 \\
\hline $\mathrm{Na}_{2} \mathrm{O}$ & 5.11 & 5.08 & 4.49 & 4.31 & 5.28 & 4.22 & 4.32 & 4.7 & 4.97 & 4.96 & 4.6 & 5.28 & 4.21 & 5.16 & 4.45 & 3.84 \\
\hline $\mathrm{K}_{2} \mathrm{O}$ & 2.82 & 1.86 & 3.16 & 3.48 & 1.46 & 3.16 & 3.88 & 3.06 & 2.69 & 2.61 & 2.34 & 1.13 & 3.79 & 1.25 & 3.59 & 3.69 \\
\hline $\mathrm{P}_{2} \mathrm{O}_{5}$ & 0.15 & 0.12 & 0.11 & 0.13 & 0.08 & 0.07 & 0.05 & 0.01 & 0.07 & 0.09 & 0.07 & 0.12 & 0.03 & 0.09 & 0.1 & 0.01 \\
\hline LOI & 0.90 & 0.80 & 1.40 & 0.70 & 1.00 & 1.30 & 1.00 & 0.70 & 0.90 & 1.00 & 1.00 & 0.60 & 0.60 & 0.50 & 0.50 & 0.90 \\
\hline Totals & 99.67 & 99.69 & 99.75 & 99.61 & 99.71 & 99.66 & 99.65 & 99.71 & 99.6 & 99.61 & 99.81 & 99.89 & 99.87 & 99.72 & 99.64 & 99.65 \\
\hline $\mathrm{Ni}$ & 7.7 & 7.5 & 12.5 & 9 & 7 & 6.5 & 6.3 & 9.8 & 8.4 & 8.8 & 8.7 & 11.37 & 6.3 & 3.6 & 5.97 & 1.34 \\
\hline Co & 5 & 5.3 & 4.1 & 4.5 & 4.7 & 3.9 & 2.6 & 2.2 & 4.2 & 4 & 3.6 & 8.28 & 1.41 & 5.05 & 4.34 & 2.62 \\
\hline $\mathrm{Rb}$ & 84.7 & 100 & 110 & 106 & 56 & 103 & 11 & 103 & 76 & 74.3 & 73.1 & 78.8 & 132 & 61.5 & 99.3 & 101 \\
\hline $\mathrm{Sr}$ & 737 & 465 & 551 & 620 & 603 & 490 & 457 & 405 & 689 & 669 & 449 & 866 & 562 & 761 & 1264 & 565 \\
\hline $\mathrm{Ba}$ & 1717 & 609 & 1131 & 1694 & 418 & 1265 & 1330 & 829 & 976 & 973 & 587 & 703 & 940 & 960 & 2156 & 2732 \\
\hline $\mathrm{Nb}$ & 7.7 & 3.47 & 5.91 & 6.54 & 3.82 & 2.13 & 2.54 & 2.89 & 3.3 & 3.33 & 5 & 5.27 & 4.22 & 4.45 & 3.6 & 2.5 \\
\hline $\mathrm{Zr}$ & 144 & 167 & 152 & 212 & 98.8 & 139 & 108 & 87.6 & 113 & 110 & 139 & 144 & 111 & 146 & 179 & 77.5 \\
\hline $\mathrm{Hf}$ & 4.1 & 3.8 & 4 & 4.8 & 2.4 & 3.5 & 3 & 2.4 & 3 & 3.1 & 3.9 & 3.22 & 3.64 & 3.65 & 4.18 & 2.58 \\
\hline Y & 13.1 & 2.7 & 9.2 & 7.9 & 3.7 & 2.4 & 3 & 2.8 & 4.2 & 4 & 5.9 & 9.8 & 5.45 & 8.74 & 7.17 & 6.02 \\
\hline $\mathrm{Pb}$ & 16.2 & 3.59 & 9.91 & 13.1 & 6.87 & 8.16 & 11.9 & 9.12 & 7.2 & 7.62 & 6.41 & 18.22 & 28.59 & 16.36 & 22.54 & 11.33 \\
\hline Th & 14.2 & 8.6 & 13.5 & 22 & 6.7 & 7.6 & 7.6 & 7.2 & 9.1 & 9.1 & 7.8 & 2.13 & 0.54 & 1.67 & 1.41 & 0.26 \\
\hline $\mathrm{La}$ & 46.3 & 38 & 45.3 & 89 & 29.4 & 32.3 & 27.9 & 18.8 & 34 & 32.8 & 25.7 & 29.92 & 21.17 & 21.54 & 55.15 & 24.24 \\
\hline $\mathrm{Ce}$ & 87.1 & 61.7 & 78.6 & 158 & 50.9 & 57.5 & 47 & 31 & 64 & 61.6 & 54 & 56.9 & 44.1 & 41.8 & 111 & 44.2 \\
\hline $\operatorname{Pr}$ & 10.3 & 6.02 & 8.63 & 16.3 & 5.67 & 5.68 & 5.03 & 3.47 & 7.21 & 7.18 & 5.29 & 7.26 & 5.22 & 5.51 & 13.99 & 4.45 \\
\hline $\mathrm{Nd}$ & 37.2 & 18.7 & 27.8 & 50.1 & 18.2 & 17.6 & 15.4 & 11 & 24.4 & 24.6 & 18.3 & 23.14 & 18.14 & 19.3 & 41.56 & 14.96 \\
\hline $\mathrm{Sm}$ & 5.9 & 2.3 & 4.1 & 5.8 & 2.4 & 2.2 & 2.2 & 1.7 & 3.1 & 3.2 & 2.9 & 5.87 & 4.44 & 4.19 & 11.08 & 4.35 \\
\hline Eu & 1.39 & 0.52 & 0.84 & 0.89 & 0.73 & 0.51 & 0.44 & 0.36 & 0.7 & 0.68 & 0.69 & 1.02 & 0.63 & 1.02 & 1.51 & 0.61 \\
\hline $\mathrm{Gd}$ & 4.79 & 1.8 & 3.66 & 4.66 & 2.02 & 1.8 & 1.78 & 1.47 & 2.28 & 2.27 & 2.45 & 2.17 & 1.86 & 2.48 & 3.09 & 1.24 \\
\hline $\mathrm{Tb}$ & 0.54 & 0.18 & 0.41 & 0.31 & 0.19 & 0.16 & 0.16 & 0.16 & 0.21 & 0.23 & 0.26 & 0.39 & 0.25 & 0.36 & 0.3 & 0.21 \\
\hline Dy & 2.55 & 0.65 & 1.89 & 1.41 & 0.87 & 0.6 & 0.7 & 0.72 & 0.93 & 0.97 & 1.32 & 1.53 & 0.7 & 1.39 & 1.21 & 1.07 \\
\hline Ho & 0.44 & 0.09 & 0.32 & 0.28 & 0.13 & 0.08 & 0.09 & 0.1 & 0.14 & 0.13 & 0.2 & 0.29 & 0.12 & 0.28 & 0.31 & 0.1508 \\
\hline Er & 1.19 & 0.27 & 0.81 & 0.58 & 0.35 & 0.27 & 0.29 & 0.28 & 0.41 & 0.37 & 0.52 & 0.54 & 0.315 & 0.77 & 0.45 & 0.2625 \\
\hline $\mathrm{Tm}$ & 0.17 & 0.05 & 0.12 & 0.09 & 0.05 & 0.05 & 0.05 & 0.05 & 0.05 & 0.05 & 0.09 & 0.11 & 0.04 & 0.08 & 0.07 & 0.03 \\
\hline $\mathrm{Yb}$ & 0.93 & 0.2717 & 0.57 & 0.52 & 0.2717 & 0.17 & 0.21 & 0.21 & 0.3 & 0.3 & 0.43 & 0.5643 & 0.1881 & 0.64 & 0.59 & 0.1672 \\
\hline $\mathrm{Lu}$ & 0.17 & 0.04 & 0.09 & 0.09 & 0.04 & 0.03 & 0.03 & 0.04 & 0.05 & 0.05 & 0.08 & 0.08 & 0.02 & 0.06 & 0.07 & 0.02 \\
\hline $\mathrm{Na}_{2} \mathrm{O}$ & 1.81 & & & 1.2 & & 1.34 & & & 1. & 1.9 & & 46 & 1.11 & 4.13 & 1.24 & 1.04 \\
\hline $\mathrm{K} / \mathrm{Rb}$ & 276.4 & 154.4 & 238.5 & 272.5 & 216.4 & 254.7 & 277.7 & 246.6 & 293.8 & 291.6 & 265.7 & 119 & 238.5 & 168.6 & 300 & 303.6 \\
\hline $\mathrm{Rb} / \mathrm{Sr}$ & 0.11 & 0.22 & 0.2 & 0.17 & 0.09 & 0.21 & 0.25 & 0.25 & 0.11 & 0.11 & 0.16 & 0.09 & 0.23 & 0.08 & 0.08 & 0.18 \\
\hline $\mathrm{Ba} / \mathrm{Rb}$ & 20.27 & 6.09 & 10.28 & 15.98 & 7.46 & 12.28 & 11.47 & & 12.84 & 13. & 8.0 & 891 & 7.13 & 15.59 & 21.7 & 27.08 \\
\hline $\mathrm{Sm} / \mathrm{Nd}$ & 0.16 & 0.12 & 0.15 & 0.12 & 0.13 & 0.13 & 0.14 & 0.15 & 0.13 & 0.13 & 0.16 & 0.25 & 0.24 & 0.22 & 0.27 & 0.29 \\
\hline $\mathrm{Sr} / \mathrm{Y}$ & 56.3 & 172.2 & 59.9 & 78.5 & 163 & 204.2 & 152.3 & 144.6 & 164 & 167.3 & 76.1 & 88.4 & 103.2 & 87 & 176.3 & 93.9 \\
\hline $\mathrm{Eu} / \mathrm{Eu}^{*}$ & 0.80 & 0.78 & 0.66 & 0.52 & 1.01 & 0.78 & 0.68 & 0.70 & 0.81 & 0.77 & 0.79 & 0.87 & 0.67 & 0.97 & 0.79 & 0.80 \\
\hline $\mathrm{La} / \mathrm{YbN}$ & 33.6 & 94.3 & 53.6 & 115.4 & 73.0 & 128.1 & 89.6 & 60.4 & 76.4 & 73.7 & 40.3 & 35.7 & 75.9 & 22.7 & 63.0 & 97.7 \\
\hline $\mathrm{Gd} / \mathrm{YbN}$ & 4.16 & 5.35 & 5.18 & 7.23 & 6.00 & 8.54 & 6.84 & 5.65 & 6.13 & 6.11 & 4.60 & 3.10 & 7.98 & 3.13 & 4.23 & 5.98 \\
\hline
\end{tabular}

* Duplicate analysis 
Maboko et al.- The origin of late archaean granitoids in the Sukumaland ...

Table 1 continued......

SUITE 2 GRANITOIDS

\begin{tabular}{|c|c|c|c|c|c|c|c|c|c|c|c|c|c|c|c|c|c|}
\hline$\overline{\text { Sample }}$ & R22 & R23 & R 24 & R 25 & R63 & KM1 & KM3 & KM3* & KM5 & KM47 & KM48 & KM52 & KM53 & KM54 & KM55 & KM56 & KM57 \\
\hline Classification & G4 & G4 & G4 & G5 & G4 & G4 & G4 & G4 & G4 & G4 & G4 & G4 & G4 & G4 & G6 & G6 & G6 \\
\hline$\overline{\mathrm{SiO}_{2}}$ & 73.13 & 71.94 & 71.46 & 60.04 & 67.60 & 74.50 & 70.68 & & 73.09 & 70.45 & 66.98 & 68.45 & 67.55 & 74.75 & 74.47 & 74.01 & 72.68 \\
\hline $\mathrm{TiO}_{2}$ & 0.20 & 0.23 & 0.28 & 0.36 & 0.40 & 0.14 & 0.23 & & 0.16 & 0.45 & 0.53 & 0.58 & 0.58 & 0.22 & 0.11 & 0.19 & 0.25 \\
\hline $\mathrm{Al}_{2} \mathrm{O}_{2}$ & 13.82 & 13.89 & 14.43 & 20.00 & 13.90 & 12.71 & 15.26 & & 14.36 & 14.89 & 15.57 & 14.19 & 14.69 & 12.83 & 13.22 & 12.74 & 13.26 \\
\hline $\mathrm{Fe}_{2} \mathrm{O}_{3}$ tot & 2.00 & 2.33 & 2.69 & 3.21 & 2.86 & 1.38 & 2.01 & & 1.33 & 2.31 & 3.37 & 3.52 & 3.63 & 1.80 & 1.02 & 1.80 & 2.29 \\
\hline $\mathrm{MnO}$ & 0.08 & 0.07 & 0.06 & 0.06 & 0.04 & 0.04 & 0.05 & & 0.04 & 0.03 & 0.05 & 0.05 & 0.05 & 0.02 & 0.03 & 0.04 & 0.06 \\
\hline $\mathrm{MgO}$ & 0.32 & 0.55 & 0.70 & 1.86 & 1.41 & 0.21 & 0.35 & & 0.22 & 0.73 & 0.70 & 0.76 & 0.78 & 0.33 & 0.15 & 0.16 & 0.29 \\
\hline $\mathrm{CaO}$ & 1.07 & 1.41 & 1.99 & 0.54 & 2.84 & 1.03 & 1.49 & & 1.15 & 2.19 & 1.90 & 2.05 & 2.08 & 1.15 & 0.93 & 0.56 & 0.72 \\
\hline $\mathrm{Na}_{2} \mathrm{O}$ & 3.82 & 3.75 & 3.93 & 5.00 & 3.20 & 3.56 & 4.33 & & 3.99 & 4.23 & 4.09 & 3.97 & 3.96 & 3.23 & 4.08 & 5.29 & 5.48 \\
\hline $\mathrm{K}_{2} \mathrm{O}$ & 4.29 & 4.20 & 3.61 & 6.85 & 4.52 & 5.55 & 4.32 & & 4.54 & 3.34 & 5.61 & 4.75 & 5.30 & 5.13 & 5.10 & 4.51 & 4.37 \\
\hline $\mathrm{P}_{2} \mathrm{O}_{5}$ & 0.07 & 0.07 & 0.08 & 0.08 & 0.11 & 0.01 & 0.04 & & 0.02 & 0.10 & 0.14 & 0.17 & 0.18 & 0.01 & 0.01 & 0.01 & 0.03 \\
\hline LOI & 0.90 & 1.30 & 0.60 & 1.70 & 3.30 & 0.70 & 1.00 & & 0.90 & 1.10 & 0.90 & 1.20 & 1.00 & 0.40 & 0.80 & 0.60 & 0.50 \\
\hline Totals & 99.70 & 99.74 & 99.83 & 99.70 & 100.18 & 99.83 & 99.76 & & 99.80 & 99.82 & 99.84 & 99.69 & 99.80 & 99.87 & 99.92 & 99.91 & 99.93 \\
\hline $\mathrm{Ni}$ & 8.80 & 8.20 & 9.40 & 8.40 & 12.90 & 5.00 & 3.27 & 2.88 & 3.33 & 5.47 & 3.83 & 3.13 & 4.37 & 0.93 & 3.33 & 1.53 & 2.98 \\
\hline $\mathrm{Co}_{0}$ & 2.90 & 3.70 & 4.60 & 5.30 & 7.60 & 1.55 & 3.17 & 2.97 & 0.90 & 4.59 & 5.49 & 5.49 & 5.62 & 1.93 & 0.31 & 1.21 & 2.83 \\
\hline $\mathrm{Rb}$ & 199 & 254 & 215 & 365 & 40 & 159 & 140 & 162 & 267 & 120 & 289 & 215 & 259 & 116 & 293 & 331 & 353 \\
\hline $\mathrm{Sr}$ & 161 & 196 & 250 & 194 & 136 & 99 & 158 & 166 & 126 & 315 & 319 & 326 & 321 & 176 & 112 & 53 & 74 \\
\hline $\mathrm{Ba}$ & 924 & 547 & 576 & 937 & 287 & 379 & 662 & 679 & 416 & 1066 & 808 & 675 & 718 & 882 & 475 & 133 & 266 \\
\hline $\mathrm{Nb}$ & 8.81 & 11.30 & 10.60 & 14.90 & 4.20 & 5.87 & 9.37 & 9.48 & 12.81 & 6.68 & 19.10 & 23.64 & 24.29 & 4.62 & 13.16 & 35.47 & 28.41 \\
\hline $\mathrm{Zr}$ & 133 & 122 & 144 & 268 & 144 & 114 & 150 & 150 & 121 & 267 & 355 & 398 & 321 & 169 & 114 & 405 & 199 \\
\hline $\mathrm{Hf}$ & 4.00 & 4.10 & 4.30 & 7.20 & 3.10 & 3.34 & 4.47 & 3.94 & 3.58 & 5.80 & 8.01 & 9.59 & 7.72 & 3.98 & 3.30 & 9.79 & 5.54 \\
\hline Y & 13.60 & 15.30 & 14.40 & 12.80 & 6.20 & 6.34 & 14.90 & 16.13 & 20.16 & 7.55 & 20.66 & 24.25 & 24.93 & 8.59 & 9.31 & 18.68 & 19.89 \\
\hline $\mathrm{Pb}$ & 14.60 & 13.20 & 12.80 & 10.40 & 2.10 & 29.02 & 20.38 & 21.73 & 29.80 & 15.26 & 28.37 & 33.55 & 24.55 & 18.84 & 24.68 & 49.35 & 30.30 \\
\hline Th & 20.40 & 20.70 & 23.30 & 36.30 & 2.80 & 0.65 & 0.98 & 1.02 & 0.72 & 2.26 & 2.54 & 2.94 & 3.19 & 0.98 & 0.48 & 1.09 & 1.24 \\
\hline $\mathrm{La}$ & 52.10 & 31.00 & 37.10 & 56.20 & 21.00 & 28.73 & 37.49 & 39.46 & 28.93 & 44.29 & 87.08 & 98.13 & 73.48 & 69.04 & 21.34 & 46.07 & 98.15 \\
\hline $\mathrm{Ce}$ & 90.7 & 55.4 & 65.5 & 96.5 & 38.0 & 51.9 & 73.1 & 75.9 & 57.4 & 83.5 & 175.5 & 206.0 & 161.5 & 135.4 & 39.2 & 100.5 & 150.8 \\
\hline $\operatorname{Pr}$ & 9.42 & 6.43 & 7.52 & 10.70 & 4.40 & 3.97 & 6.41 & 6.71 & 5.04 & 7.17 & 16.62 & 19.08 & 16.14 & 12.48 & 2.58 & 6.87 & 12.00 \\
\hline $\mathrm{Nd}$ & 29.90 & 21.90 & 24.30 & 33.00 & 17.00 & 17.38 & 27.37 & 28.99 & 22.62 & 25.28 & 57.39 & 68.50 & 57.85 & 43.59 & 12.74 & 22.27 & 39.23 \\
\hline Sm & 4.40 & 3.90 & 3.90 & 4.90 & 2.50 & 5.05 & 7.53 & 7.67 & 5.66 & 7.69 & 17.34 & 20.33 & 16.05 & 13.38 & 3.73 & 8.69 & 14.85 \\
\hline Eu & 0.66 & 0.61 & 0.75 & 0.57 & 0.78 & 0.45 & 0.72 & 0.81 & 0.41 & 0.70 & 1.34 & 1.35 & 1.45 & 1.05 & 0.30 & 0.45 & 0.72 \\
\hline $\mathrm{Gd}$ & 3.90 & 3.31 & 3.27 & 3.91 & 2.20 & 1.67 & 3.53 & 3.65 & 4.51 & 3.14 & 6.09 & 6.51 & 6.05 & 3.12 & 2.73 & 6.81 & 5.45 \\
\hline $\mathrm{Tb}$ & 0.46 & 0.46 & 0.46 & 0.49 & 0.20 & 0.25 & 0.40 & 0.39 & 0.43 & 0.31 & 0. & 0.38 & 0.30 & 0.39 & 0.23 & 0.33 & 0.38 \\
\hline Dy & 2.24 & 2.51 & 2.36 & 2.38 & 1.30 & 1.51 & 2.38 & 2.56 & 2.75 & 2.12 & 3.25 & 3.71 & 3.74 & 1.47 & 1.28 & 2.08 & 2.85 \\
\hline Ho & 0.44 & 0.48 & 0.49 & 0.46 & 0.20 & 0.32 & 0.37 & 0.37 & 0.42 & 0.48 & 0.58 & 0.72 & 0.63 & 0.32 & 0.27 & 0.34 & 0.49 \\
\hline $\mathrm{Er}$ & 1.22 & 1.27 & 1.27 & 1.27 & 0.50 & 0.84 & 0.80 & 0.82 & 0.89 & 1.09 & 1.47 & 1.43 & 1.49 & 0.80 & 0.50 & 1.05 & 1.07 \\
\hline $\mathrm{Tm}$ & 0.20 & 0.24 & 0.20 & 0.16 & 0.10 & 0.13 & 0.13 & 0.13 & 0.13 & 0.10 & 0.14 & 0.15 & 0.15 & 0.14 & 0.07 & 0.13 & 0.13 \\
\hline $\mathrm{Yb}$ & 1.12 & 1.36 & 1.28 & 0.79 & 0.50 & 0.78 & 0.69 & 0.69 & 0.79 & 0.57 & 0.73 & 0.88 & 0.86 & 0.81 & 0.44 & 0.82 & 0.77 \\
\hline $\mathrm{Lu}$ & 0.21 & 0.25 & 0.24 & 0.12 & 0.10 & 0.09 & 0.10 & 0.10 & 0.13 & 0.07 & 0.11 & 0.12 & 0.13 & 0.12 & 0.06 & 0.13 & 0.12 \\
\hline $\mathrm{Na}_{2} \mathrm{O} / \mathrm{K}_{2} \mathrm{O}$ & 0.90 & 0.90 & 1.10 & 0.70 & 0.71 & 0.60 & 1.00 & 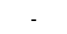 & 0.90 & 1.30 & 0.70 & 0.80 & 0.70 & 0.60 & 0.80 & 1.20 & 1.30 \\
\hline $\mathrm{K} / \mathrm{Rb}$ & 179 & 137 & 139 & 156 & 466 & 289 & 256 & 221 & 141 & 232 & 161 & 183 & 170 & 367 & 145 & 113 & 103 \\
\hline $\mathrm{Rb} / \mathrm{Sr}$ & 1.24 & 1.30 & 0.86 & 1.88 & 0.30 & 1.62 & 0.89 & 0.98 & 2.12 & 0.38 & 0.91 & 0.66 & 0.81 & 0.66 & 2.62 & 6.27 & 4.81 \\
\hline $\mathrm{Ba} / \mathrm{Rb}$ & 4.64 & 2.15 & 2.68 & 2.57 & 2.11 & 2.38 & 4.72 & 4.19 & 1.56 & 8.91 & 2.80 & 3.14 & 2.77 & 7.60 & 1.62 & 0.40 & 0.75 \\
\hline $\mathrm{Sm} / \mathrm{Nd}$ & 0.15 & 0.18 & 0.16 & 0.15 & 0.15 & 0.29 & 0.28 & 0.26 & 0.25 & 0.30 & 0.30 & 0.30 & 0.28 & 0.31 & 0.29 & 0.39 & 0.38 \\
\hline $\mathrm{Sr} / \mathrm{Y}$ & 11.80 & 12.80 & 17.40 & 15.20 & 21.94 & 15.50 & 10.60 & 10.30 & 6.30 & 41.70 & 15.40 & 13.40 & 12.90 & 20.50 & 12.00 & 2.80 & 3.70 \\
\hline $\mathrm{Eu} / \mathrm{Eu}^{*}$ & 0.49 & 0.52 & 0.64 & 0.40 & 1.02 & 0.47 & 0.43 & 0.47 & 0.25 & 0.44 & 0.40 & 0.36 & 0.45 & 0.50 & 0.29 & 0.18 & 0.24 \\
\hline $\mathrm{La} / \mathrm{YbN}$ & 31.4 & 15.4 & 19.5 & 47.7 & 28.3 & 24.8 & 36.6 & 38.6 & 24.6 & 52.4 & 80.3 & 75.4 & 57.8 & 57.5 & 32.8 & 38.1 & 85.6 \\
\hline $\mathrm{Gd} / \mathrm{YbN}$ & 2.81 & 1.96 & 2.06 & 3.97 & 3.55 & 1.73 & 4.13 & 4.27 & 4.58 & 4.45 & 6.72 & 5.98 & 5.70 & 3.11 & 5.02 & 6.74 & 5.69 \\
\hline
\end{tabular}

* Duplicate analysis

The second suite consists of 16 samples which are characterised by $\mathrm{Na}_{2} \mathrm{O} / \mathrm{K}_{2} \mathrm{O}<1$ (except samples R24, KM3, KM47, KM56 and KM57, which have ratios between 1.10 and 1.30). On the Ab-An-Or diagram, Suite
2 rocks plot mainly in the granite field except for one sample that plots in the trondhjemite field (Fig. 2). Compared to Suite 1 , Suite 2 rocks have lower $\mathrm{Na}_{2} \mathrm{O}$ $($ mean $=4.12 \mathrm{wt} \%)$ and higher $\mathrm{K}_{2} \mathrm{O}$ (mean 
$=4.65 \mathrm{wt} \%$ ) contents. They are also characterised by low $\mathrm{Sr} / \mathrm{Y}(2.80-41.7$, mean $=14.4)$, low $\mathrm{Ba} / \mathrm{Rb}(0.40-8.91$, mean $=3.23)$ and high $\mathrm{Rb} / \mathrm{Sr}(0.30-6.27$, mean $=$ 1.67) ratios. Compared to Suite 1 , these rocks also have low $\mathrm{Sr}$ (53 - $326 \mathrm{ppm}$, mean
$=187$ ppm), high $\mathrm{Rb}(40-365$ ppm, mean = $222 \mathrm{ppm})$ and high $\mathrm{Yb}(0.44-1.36 \mathrm{ppm}$, mean $=0.82 \mathrm{ppm})$ contents. $\mathrm{K} / \mathrm{Rb}$ ratios range between 103 and $466($ mean $=203)$ and are generally lower than in the suite 1 samples.

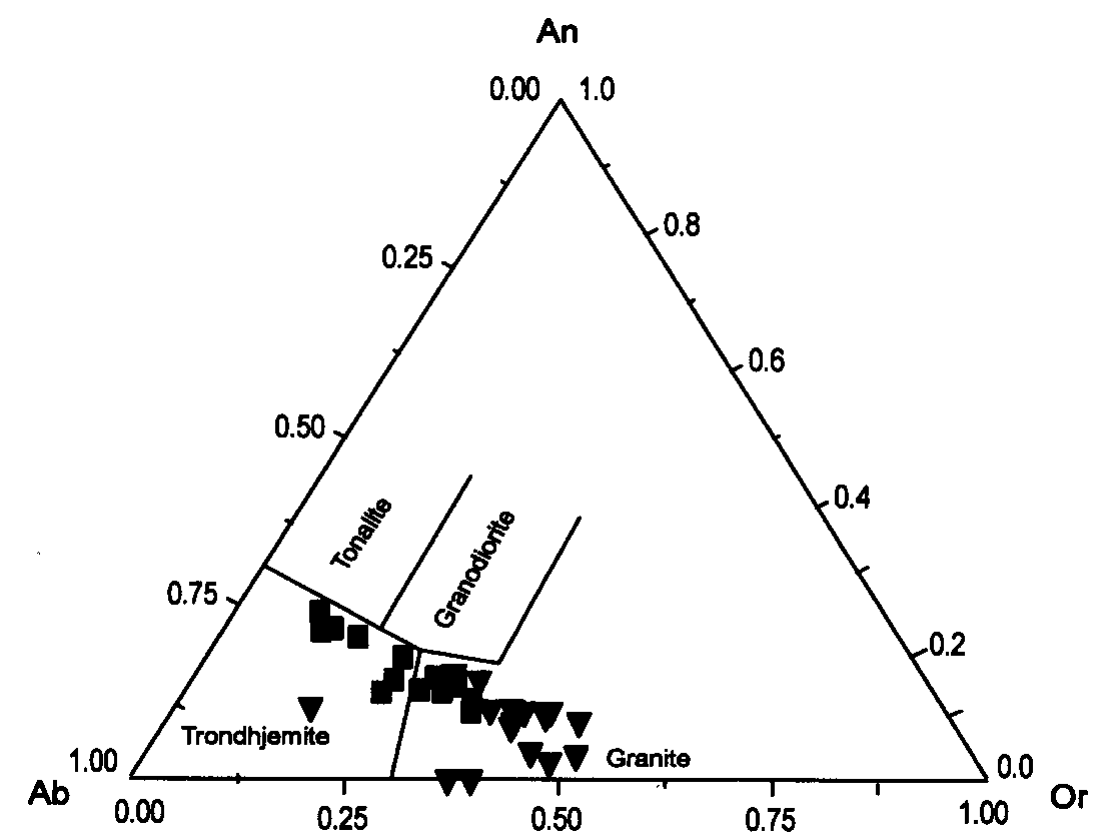

Figure 2: Classification of the SGB Suites 1 (filled triangles) and 2 (filled triangles) granitoids according to their molecular An-Ab-Or composition. Note the granitic to trondhjemitic character. The field boundaries are as defined by Barker (1979).

Suite 2 rocks generally display less steep chondrite-normalised REE patterns (Fig. 5). $(\mathrm{La} / \mathrm{Yb})_{\mathrm{N}}$ values range between 15 and 86 with a mean value of 44 , significantly lower than the corresponding Suite 1 values. The rocks are characterised by higher concentrations of the HREE compared to Suite 1 samples as revealed by $\mathrm{Yb}_{\mathrm{N}}$ values of between 2.1 and 6.5 (mean 3.90). On primitive mantle-normalised spidergrams, Suite 2 samples, like those of Suite 1 show relative depletion in $\mathrm{Th}, \mathrm{Nb}$ and $\mathrm{Ti}$, together with $\mathrm{P}$ and $\mathrm{Y}$ whereas $\mathrm{Zr}$ and $\mathrm{Hf}$ are enriched relative to adjacent elements. Unlike Suite 1 samples, however, Suite 2 rocks are depleted in $\mathrm{Sr}$ relative to $\mathrm{Ce}$ and Nd (Fig. 6). 


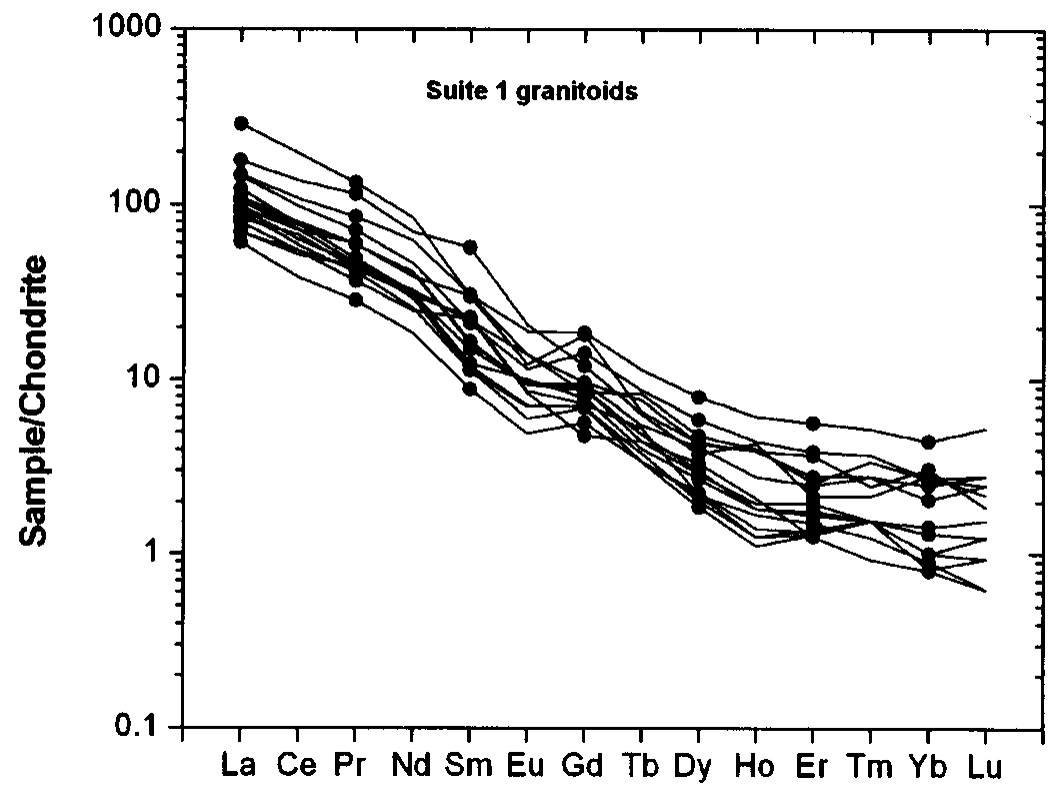

Figure 3: Chondrite-normalised REE diagrams for the Suite 1 granitoids.

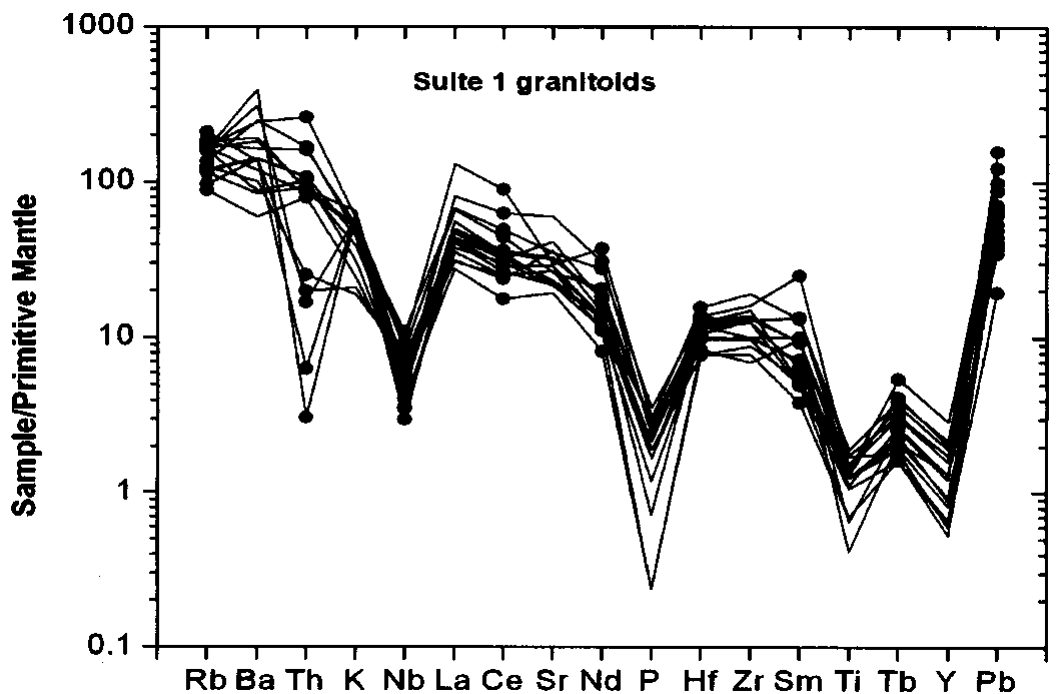

Figure 4: Primitive Mantle-normalised spidergrams for the Suite 1 granitoids. 


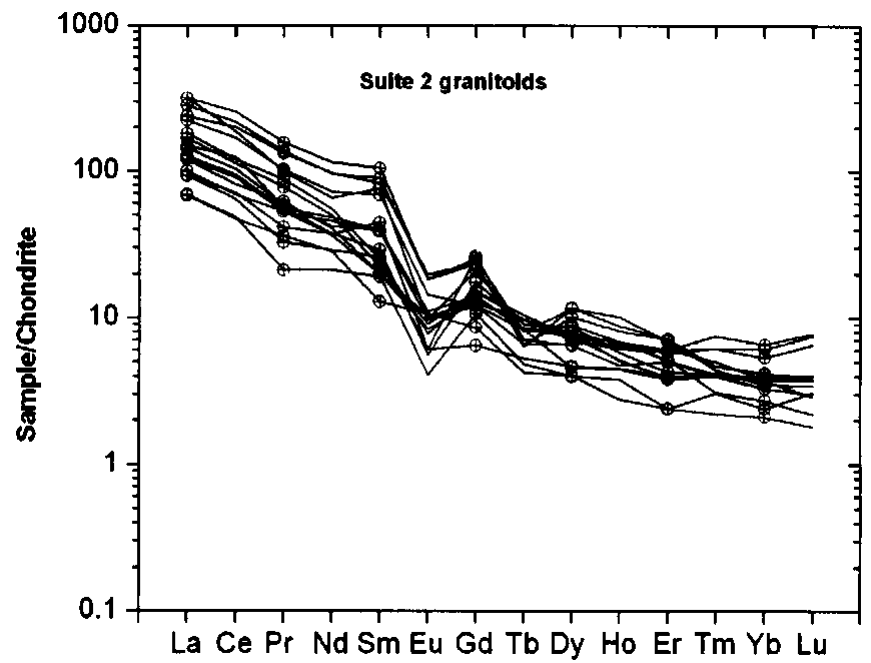

Figure 5: Chondrite-normalised REE diagrams for the Suite 2 granitoids.

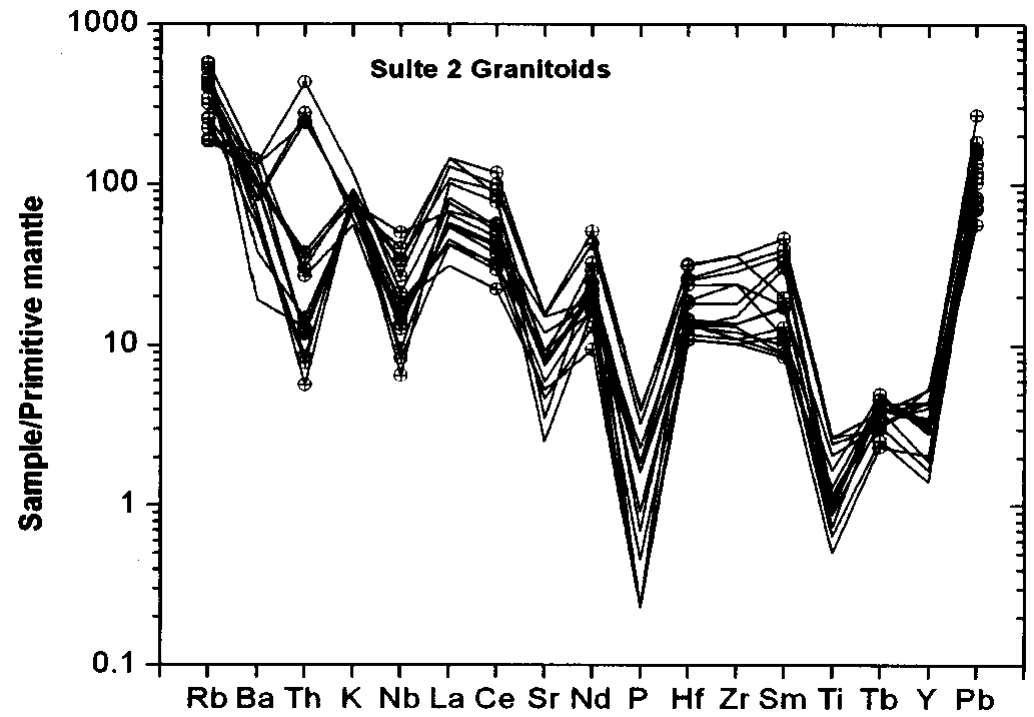

Figure 6: Primitive Mantle-normalised spidergrams for the Suite 2 granitoids.

Emplacement and crustal formation ages

$\mathrm{Nd}$ isotopic data from 15 samples (10 from Suite 1 and 5 from Suite 2 granitoids) are presented in Table 2. Also shown in Table 2 are $\mathrm{Nd}$ depleted mantle mean crustal residence ages $\left(\mathrm{T}_{\mathrm{DM}}\right)$ calculated assuming a linear evolution model for the mantle together with present day ${ }^{143} \mathrm{Nd} /{ }^{144} \mathrm{Nd}$ and ${ }^{147} \mathrm{Sm} /{ }^{144} \mathrm{Nd}$ mantle values of 0.513114 and
0.222, respectively (Michard et al. 1985). The $\mathrm{T}_{\mathrm{DM}}$ ages range between 2470 and 2710 Ma with a mean of $2610 \pm 35$ Ma (2 SE). Nine samples (R15, R16, R17, R18, R19, R20 from Suite 1; R23, R24 and R63 from Suite 2), however, define a more restricted $\mathrm{T}_{\mathrm{DM}}$ range, between 2610 and $2650 \mathrm{Ma}$, with a mean of $2632 \pm 11 \mathrm{Ma}$. The mean $\mathrm{T}_{\mathrm{DM}}$ age 
Maboko et al.- The origin of late archaean granitoids in the Sukumaland ...

of $2610 \pm 35 \mathrm{Ma}$ is indistinguishable from the $\mathrm{Rb}-\mathrm{Sr}$ isochron age of $2620 \pm 30 \mathrm{Ma}$ $\left(\left({ }^{87} \mathrm{Sr} /{ }^{86} \mathrm{Sr}\right)_{\text {initial }}=0.7017 \pm 4\right)$ previously obtained by Bell and Dodson (1981) for granitoids from the Geita area. Calculated initial $\varepsilon_{\mathrm{Nd}}$ values assuming a $2620 \mathrm{Ma}$ emplacement age range between -0.66 and 3.58 but cluster between 0.28 and 0.84 (mean $=0.53 \pm 0.11$ ) for the samples yielding a tight cluster in $\mathrm{T}_{\mathrm{DM}}$ ages. The remaining 6 samples can be divided into two groups: the two Suite 1 samples, R2 and $\mathrm{R} 21$, yield negative $\varepsilon_{\mathrm{Nd}}$ values $(-0.66$ and 0.80 , respectively) and $\mathrm{T}_{\mathrm{DM}}$ ages of 2708 and 2703 Ma. The latter are slightly older than the $\sim 2630 \mathrm{Ma}$ age of the 9 samples, which define a tight $\mathrm{T}_{\mathrm{DM}}$ cluster. The remaining four samples (R14, R22, 25 and $\mathrm{R} 26)$ yield $\varepsilon_{\mathrm{Nd}}$ values of between 1.69 and 3.58 and $\mathrm{T}_{\mathrm{DM}}$ ages of between 2470 and $2570 \mathrm{Ma}$, younger than the samples with mean crustal residence ages of $\sim 2630 \mathrm{Ma}$.
The similarity of the emplacement age to the mean crustal residence ages indicates that these granitoids represent late Archaean juvenile additions to the continental crust. The juvenile nature of the granitoids is also consistent with the low initial ${ }^{87} \mathrm{Sr} /{ }^{86} \mathrm{Sr}$ ratio $(0.7017 \pm 4)$. Samples R2 and R21 which yield negative $\varepsilon_{\mathrm{Nd}}$ values $(-0.66$ and -0.80 respectively) and slightly older $\mathrm{T}_{\mathrm{DM}}$ ages (2708 and $2703 \mathrm{Ma}$ ) may indicate slight contamination of $\sim 2640$ Ma juvenile granitoid melt by older crustal material. On the other hand, the four samples (R14, R22, 25 and R26) which yield $\varepsilon_{\mathrm{Nd}}$ values between 1.69 and 3.58 and $\mathrm{T}_{\mathrm{DM}}$ ages of between 2470 and $2570 \mathrm{Ma}$ may indicate derivation from a slightly younger protolith. Samples R14 and R26 belong to geochemical Suite 1 whereas samples R22 and R25 belong to Suite 2 suggesting that there is no significant difference in the mean crustal residence ages of the two suites. This is also true for the nine samples that define a tighter cluster in $\mathrm{T}_{\mathrm{DM}}$ ages.

Table 2: $\quad$ Sm-Nd isotopic data for granitoids from the Sukumaland Greenstone Belt

\begin{tabular}{cccccccc}
\hline Sample & Classification & $\begin{array}{c}\mathrm{Nd} \\
(\mathrm{ppm})\end{array}$ & $\begin{array}{c}\mathrm{Sm} \\
(\mathrm{ppm})\end{array}$ & ${ }^{143} \mathrm{Nd} /{ }^{144} \mathrm{Nd}$ & ${ }^{147} \mathrm{Sm} /{ }^{144} \mathrm{Nd}$ & $\mathrm{T}_{\mathrm{DM}}$ & $\varepsilon(2.62)$ \\
\hline R 2 & G3, Suite 1 & 36.07 & 5.95 & $0.510921 \pm 7$ & 0.099 & 2708 & -0.66 \\
R 14 & G3, Suite 1 & 24.4 & 3.26 & $0.510810 \pm 6$ & 0.08 & 2467 & 3.58 \\
R 15 & G3, Suite 1 & 32.81 & 5.12 & $0.510875 \pm 6$ & 0.094 & 2648 & 0.28 \\
R 16 & G3, Suite 1 & 44.67 & 5.44 & $0.510531 \pm 6$ & 0.073 & 2634 & 0.48 \\
R 17 & G3, Suite 1 & 15.88 & 2.14 & $0.510658 \pm 7$ & 0.081 & 2640 & 0.38 \\
R 18 & G3, Suite 1 & 18.51 & 2.24 & $0.510527 \pm 7$ & 0.073 & 2627 & 0.62 \\
R 19 & G3, Suite 1 & 12 & 1.67 & $0.510729 \pm 7$ & 0.084 & 2621 & 0.74 \\
R 20 & G3, Suite 1 & 13.86 & 2.11 & $0.510845 \pm 7$ & 0.092 & 2638 & 0.44 \\
R 21 & G3, Suite 1 & 21.07 & 2.76 & $0.510560 \pm 6$ & 0.079 & 2703 & -0.8 \\
R 26 & G3, Suite 1 & 27.27 & 4.6 & $0.511154 \pm 6$ & 0.101 & 2466 & 3.17 \\
& & & & & & & 2566 \\
R 22 & G4, Suite 2 & 24.45 & 3.64 & $0.510874 \pm 7$ & 0.09 & 1.69 \\
R 23 & G4, Suite 2 & 16.08 & 2.83 & $0.511115 \pm 6$ & 0.106 & 2614 & 0.84 \\
R 24 & G4, Suite 2 & 24.87 & 4.11 & $0.510990 \pm 7$ & 0.1 & 2630 & 0.6 \\
R 25 & G5, Suite 2 & 31.6 & 4.69 & $0.510878 \pm 5$ & 0.089 & 2554 & 1.89 \\
R 63 & G4, Suite 2 & 14.46 & 2.22 & $0.510857 \pm 7$ & 0.092 & 2641 & 0.4 \\
\hline
\end{tabular}




\section{DISCUSSION}

The relatively low $\mathrm{Rb}$ contents even at high $\mathrm{SiO}_{2}$ values suggest that both Suite 1 and Suite 2 rocks crystallised from little differentiated magmas. Granitoids with a major element composition similar to the Geita rocks can be generated by partial melting of hydrated basalt which has been transformed into eclogite or garnet amphibolite (Arth and Hanson 1972, Drummond and Defant 1990, Rapp et al. 1991, Martin 1999, Smithies 2000). Experimental studies further indicate that partial melting of hydrated low K-tholeiite under water undersaturated conditions can produce high $\mathrm{Al}_{2} \mathrm{O}_{3}$ granitoids, similar to the Geita rocks, over a wide range of PT conditions leaving a hornblende and garnetrich residue (Wolf and Wyllie 1989, Rapp et al. 1991, Rapp and Watson 1995). The $\mathrm{Al}_{2} \mathrm{O}_{3}$ rich nature of the samples can, therefore, be used to infer their formation by partial melting of hydrated basalt at pressures sufficiently high to stabilise hornblende as a residue phase. Melting in the presence of hornblende as a residue phase can also explain the low to moderate $\mathrm{K} / \mathrm{Rb}$ ratios $(137-360)$ because of the greater affinity of hornblende for $\mathrm{K}$ relative to $\mathrm{Rb}$ (Arth and Hanson 1972). In the absence of hornblende, melting would produce a liquid which is highly enriched in $\mathrm{K}$ resulting in granitoids with high $\mathrm{K} / \mathrm{Rb}$ ratios (>550 - 650, Drummond and Defant 1990).

The highly fractionated REE patterns as well as the low Y contents further indicate the presence of residual garnet in the sources of both Suites 1 and 2 (Martin 1999). The lower Y content of Suite 1 samples relative to that of Suite 2 most likely reflects relatively higher modal abundance of garnet in its source. The high $\mathrm{Sr}$ contents of Suite 1 rocks $($ mean $=616 \mathrm{ppm})$, high $\mathrm{Ba}($ mean $=$ $1079 \mathrm{ppm}$ ), coupled with the lack of significant Eu anomalies (mean $\mathrm{Eu}^{*}=0.8$ ) suggests that plagioclase fractionation was not an important process. This suggests an origin of the Suite 1 granitoids by partial melting of hydrated basalt in the absence of plagioclase as a residue phase. This suggests formation of the granitoids by partial melting of a hornblende eclogite rather than an amphibolite.

In contrast to Suite 1 rocks, the Suite 2 rocks are characterised by lower $\mathrm{Sr}$ (mean $=174$ $\mathrm{ppm})$ and $\mathrm{Ba}($ mean $=587 \mathrm{ppm})$ contents as well as prominent negative Eu anomalies (mean $=0.5)$ suggesting significant fractionation of plagioclase during their petrogenesis. Given the relatively low $\mathrm{Rb}$ contents even at high $\mathrm{SiO}_{2}$ values indicative of crystallisation of the rocks from little differentiated magmas, this suggests an origin of the Suite 2 granitoids by partial melting of a source in which plagioclase was a residual phase. This further indicates that the rocks were formed by partial melting of garnet amphibolite rather than hornblende eclogite. As amphibolites tend to have lower modal abundances of garnet than eclogites, formation by partial melting of amphibolite also explains the relatively higher abundance of $\mathrm{Y}$ and other HREE in Suite 2 samples. 


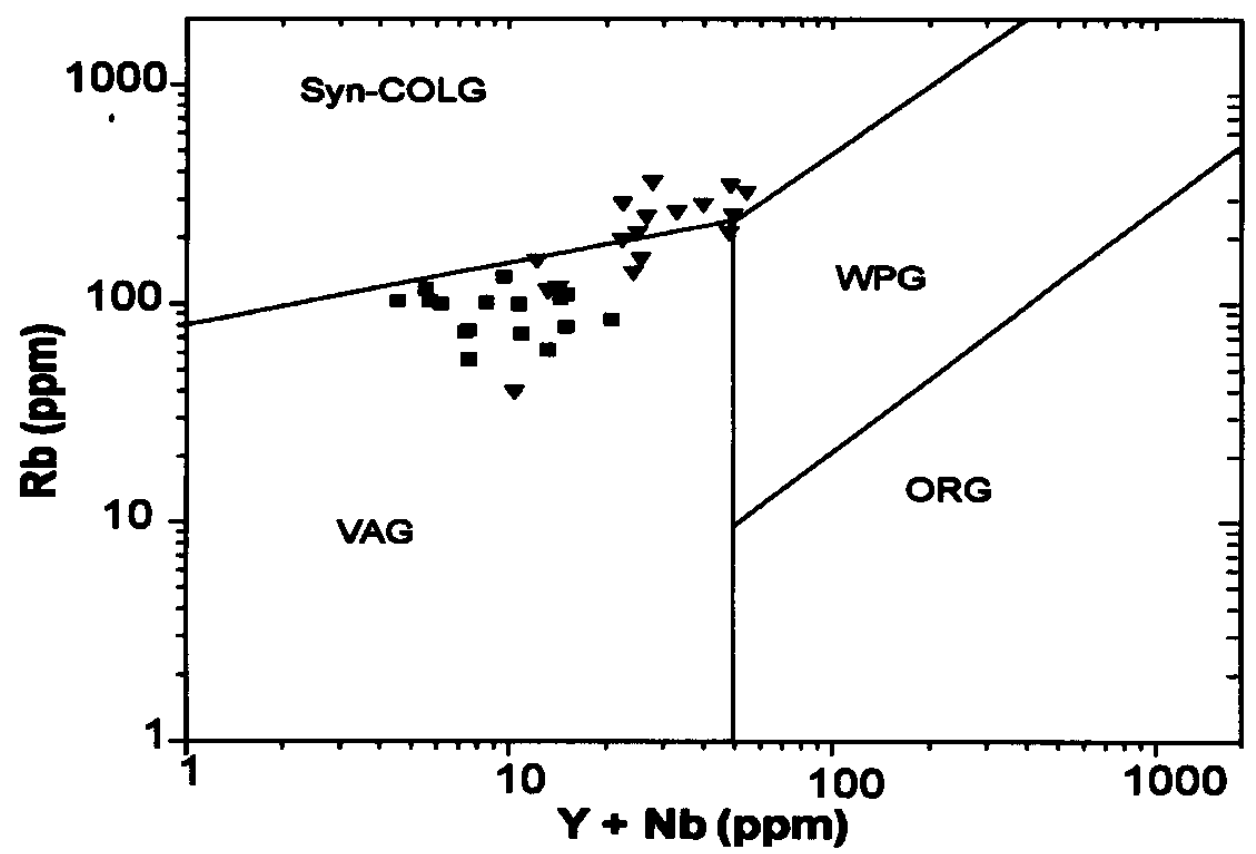

Figure 7: $\quad \mathrm{Rb}$ versus $\mathrm{Y}+\mathrm{Nb}$ discrimination diagrams (After Pearce et al. 1984) for the SGB Suites 1 (filled squares) and Suite 2 (filled triangles) granitoids.

\section{Geodynamic setting of melting}

The samples are plotted on the $\mathrm{Rb}$ versus $\mathrm{Y}+\mathrm{Nb}$ tectonic discrimination diagram of Pearce et al. (1984) on Fig. 7. All Suite 1 samples plot as volcanic arc granites whereas most of the Suite 2 samples plot as syn-collisional granites with a few plotting in the volcanic arc field. This, together with the prominent negative $\mathrm{Nb}$ and $\mathrm{Ti}$ anomalies which are characteristic of both Suites 1 and 2 suggest a convergent margin setting for the granitic magmatism in the Sukumaland Greenstone Belt. The similarity of the mean crustal residence ages of the two suites of granitoids further indicates that the magmatism occurred at more or less the same time with the main geochemical difference between Suite 1 and Suite 2 being the depth of melting. To explain the convergent margin setting as well as the over all geochemical characteristics of the rocks, it is proposed that the two suites formed by partial melting at the base of a thickened sub-arc basaltic crust composed of the Nyanzian tholeiites. Melting to form the Suite 1 granitoids occurred at greater depth in the eclogite stability field whereas Suite 2 samples formed by melting at shallower depth in the garnet amphibolite stability field.

\section{ACKNOWLEDEMENTS}

This work was funded by Sida/SAREC through the Research Capacity Development in the Faculty of Science Project (Geoscience sub-project). We wish to acknowledge the assistance of Charles Messo with the geochemical analyses at Dar es Salaam.

\section{REFERENCES}

Arth JG and Hanson GN 1972 Quartz diorites derived by partial melting of eclogite or amphibolite at mantle depths. Contrib. Miner. Petrol. 37: 61174. 
Atherton MP and Petford N 1993 Generation of sodium-rich magmas from newly underplated basaltic crust. Nature 362: 144-146.

Barker F 1979 Trondhjemites: Definition, environment and hypotheses of origin. In: Barker F (ed) Trondhjemites, dacites and related rocks. Elsevier, Amsterdam, pp. 1-12.

Barth H 1990 Provisional Geological Map of the Lake Victoria Gold Fields, Tanzania 1: 500000 (with explanatory notes). Geol. Jb. B72, pp. 3-59.

Bell K and Dodson MH 1981 The geochronology of the Tanzanian Shield. J. Geol. 89: 109-228.

Borg G and Krogh T 1999 Isotopic age data of single zircons from the Archaean Sukumaland Greenstone Belt, Tanzania. J. Afr. Earth Sci. 29: 301312 .

Borg G and Shackleton RM 1997 The Tanzania and NE Zaire Cratons. In: de Wit MJ and Ashwal LD (eds). Greenstone Belts. Clarendon Press, Oxford, pp. 608-619.

Borg G, Lyatuu DR and Rammlmair D 1990 Genetic aspects of the Geita and Jubilee Reef Archaean BIF-hosted gold deposits, Tanzania. Geol. Rundschau 79: $355-371$.

Boynton WV 1984 Cosmochemistry of the rare earth elements: Meteorite studies. In: Henderson P (ed) Rare Earth Element Geochemistry, Elsevier, Amsterdam, pp. 63-107.

Drummond MS and Defant MJ 1990 A model for trondhjemite-tonalite-dacite genesis and crustal growth via slab melting: Archaean to modern comparison. J. Geophys. Res. 95: 21503-21521.

Grantham DR, Temperley BN and McConnell RB 1945 Explanation of the geolgy of degree sheet No.17, Kahama. Bull. Geol. Surv. Tanganyika, Dar es Salaam, 39 pp.

Manya S and Maboko MAH 2003 Dating basaltic volcanism in the Neoarchaean Sukumaland Greenstone Belt of the
Tanzania Craton using the Sm-Nd method: implications for the geological evolution of the Tanzania Craton. Precam. Res. 121: 35-45.

Martin H 1999 Adakitic magmas: modern analogues of Archaean granitoids. Lithos 46: 411-429.

Martin H, Smithies RH, Rapp R, Moyen JF and Champion D 2005 An overview of adakite, tonalite-trondhjemitegranodiorite (TTG), and sanukitoid: relationship and some implications for crustal evolution. Lithos 79: 1-24.

McDonough WF and Sun SS 1995 The composition of the Earth. Chemical Geology 120: 223-253.

Messo CW 2004 Geochemistry of Neoarchaean volcanic rocks of the Ikoma area in the Kilimafedha greenstone belt, northwest Tanzania. M.Sc. Thesis. University of Dar es Salaam.

Michard A, Gurriet P, Soudant M and Albarede F 1985 Nd isotopes in French Phanerozoic shales: external vs internal aspects of crustal evolution. Geochim. Cosmochim. Acta 49: 601-610.

Pearce JA, Harris NBW and Tindle GA 1984 Trace element discrimination diagrams for the tectonic interpretation of granitic rocks. J. Petrol. 25: 956983.

Rapp RP and Watson EB 1995 Dehydration melting of metabasalt at $8-32 \mathrm{~kb}$ : implications for continental growth and crust-mantle recycling. J. Petrol. 36: 891-931.

Rapp RP, Watson EB and Miller CF 1991 Partial melting of amphibolite/eclogite and the origin of Archaean trondhjemites and tonalites. Precam. Res. 51: 1-25.

Richard P, Shimizu N and Allegre CJ 1976 ${ }^{143} \mathrm{Nd} /{ }^{146} \mathrm{Nd}$, a natural tracer: an application to oceanic basalts. Earth Planet. Sci. Lett. 31: 269-278.

Smithies RH 2000 The Archaean tonalitetrondhjemite-granodiorite (TTG) series is not an analogue of Cenozoic adakite. Earth Planet. Sci. Lett. 182: 15-125. 
Maboko et al.- The origin of late archaean granitoids in the Sukumaland ...

Wolf MB and Wyllie PJ 1989 The formation of tonalitic liquids during the vapour- absent partial melting of amphibolite at $10 \mathrm{~kb}$. Eos 70, 506. 